\title{
La synthèse de textes dans l'nseignement supérieur, un objet à fort enjeu local : analyse comparative dans deux institutions, l'ENA et l'IUFM
}

The summarising of texts in higher education, an objective which raises significant issues: a comparative analysis of two institutions, E.N.A. and the I.U.F.M

\section{(2) OpenEdition}

\section{Journals}

Édition électronique

URL : https://journals.openedition.org/educationdidactique/795

DOI : 10.4000/educationdidactique.795

ISBN : 978-2-7535-1624-3

ISSN : 2111-4838

\section{Éditeur}

Presses universitaires de Rennes

\section{Édition imprimée}

Date de publication : 1 octobre 2010

Pagination : 41-55

ISBN : 978-2-7535-1300-6

ISSN : 1956-3485

\section{Référence électronique}

Jean-Michel Perez, « La synthèse de textes dans I'nseignement supérieur, un objet à fort enjeu local analyse comparative dans deux institutions, I'ENA et l'IUFM », Éducation et didactique [En ligne], 4-2 I 2010, mis en ligne le 01 avril 2012, consulté le 25 avril 2022. URL : http://journals.openedition.org/ educationdidactique/795; DOI : https://doi.org/10.4000/educationdidactique.795 


\title{
LA SYNTHÈSE DE TEXTES DANS L'NSEIGNEMENT SUPÉRIEUR, UN OBJET À FORT ENJEU LOCAL : ANALYSE COMPARATIVE DANS DEUX INSTITUTIONS, L'ENA ET L'IUFM
}

\author{
Jean-Michel Pérez (ADEF, Universite d'Aix-Marseille 1)
}

\begin{abstract}
Résumé : Le présent texte s'insère dans une recherche en cours sur les exercices de "synthèse de textes » (Sy) proposés dans l'enseignement supérieur français. En faisant référence au modèle de «l'écologie des savoirs » cet article se donne pour objectif d'analyser la fonction qu'occupe Sy dans deux habitats institutionnels où il est enjeu didactique : le concours d'entrée à l'Ecole Nationale de l'Administration (ENA) et le Concours de recrutement des Professeurs des Ecoles dans les Instituts Universitaires de Formation des Maîtres (IUFM). Lanalyse montre que c'est un objet ancillaire, à fort enjeu local n'ayant pas dans les deux institutions, les mêmes contraintes ni les mêmes conditions de viabilité. Mais au-delà d'un écosystème local se dégage une écologie sociale (une niche) où le savoir peut être défini comme une compétence pour répondre à des questions à partir d'un corpus donné.
\end{abstract}

Mots clés : Approche comparée - Enseignement Supérieur - Synthèse de textes - Ecologie - Habitat-Niche.

Jean-Michel Pérez

\section{Introduction}

Cette recherche s'inscrit dans le cadre général de la didactique de l'enseignement supérieur et porte sur la « synthèse de textes » à l'Université ${ }^{1}$. S'intéresser à cet objet du point de vue du questionnement didactique pose quelques interrogations dont il faut d'emblée situer les enjeux car sa programmation est à la fois récente dans l'enseignement supérieur et d'un genre encore mal défini.

Récente, car, préalablement aux années 1971 et sous quelle que forme que ce soit la « note de synthèse » ou "synthèse de textes » ou encore « synthèse de dossier » est absente du système d'enseignement français. En effet, alors qu'elle semble faire partie du système éducatif depuis toujours, nos investigations réalisées de l'origine de l'Université à aujourd'hui, n'ont pas permis d'identifier ses formes originaires.

Elle se découvre finalement comme un exercice récemment introduit dans l'enseignement supérieur, inventée lors de la grande réforme du concours d'entrée de l'Ecole Nationale de l'Administration ${ }^{2}$. Son emploi s'est ensuite largement généralisé pour le recrutement de nombreux établissements. De ce fait, c'est un " objet », qui entre dans le système d'enseignement général, par la voie des « concours» de recrutement des institutions professionnelles de la Fonction Publique d'Etat ${ }^{3}$.

Ainsi, les exercices de "synthèse de textes » se repèrent principalement entre la fin du premier cycle universitaire -L3-, et avant l'entrée à 117 établissements de l'Enseignement Supérieur Français Public (Pérez, 2008). Ils sont programmés dans la phase dite « d'admissibilité » des concours.

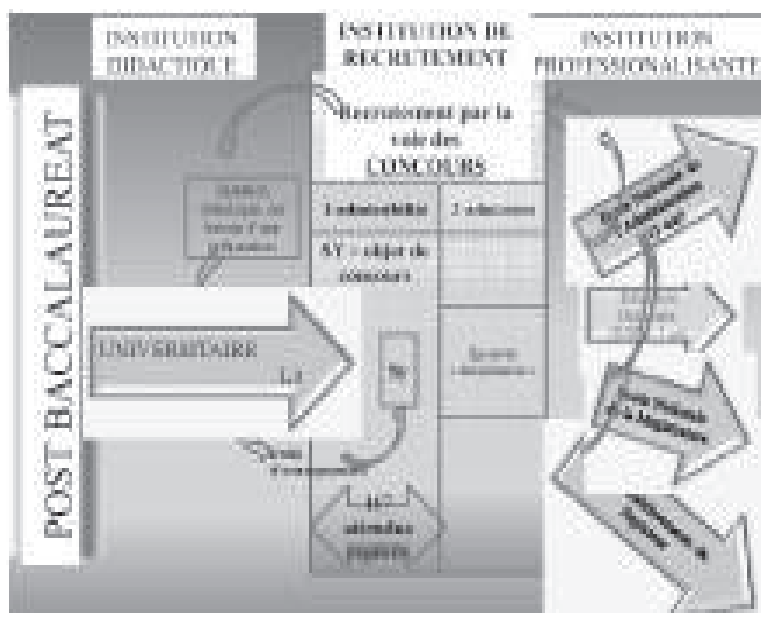

Espace où il se repère des synthèses écrites, que nous nommons Sy dans l'Enseignement Supérieur: objet de concours. 
Ces observations tendent à montrer que la « synthèse de textes » prend une place originale, par rapport aux objets issus des formes modernes de la transmission des savoirs ${ }^{4}$. Par ailleurs, si nous l'interrogeons par l'entrée disciplinaire, nous nous apercevons qu'elle est également difficile à saisir. En effet, une recherche portant sur l'ensemble du système d'enseignement, montre aussi sa quasi absence dans les instructions des Bulletins Officiels de toutes disciplines confondues (Pérez, 2008).

Elle se situerait entre la philosophie, les mathématiques et le français. Les philosophes et les mathématiciens donnent un cadre pour penser cet objet, car ils sont les seuls originairement à s'y intéresser, notamment dans le «Discours de la Science ». Mais cela reste une approche générale qu'il faut parvenir à transférer à des situations spécifiques. Et l'inscrire dans le cadre du français pose problème. En effet, de nombreux auteurs s'accordent pour dire, désormais, que sa présence est nouvelle dans l'histoire de l'enseignement : J.-P. Bernié décrit un objet « invertébré » et A. Chervel a une réflexion pouvant être utile au débat. Son ouvrage, développant et regroupant l'essentiel des recherches menées sur « l'histoire de l'enseignement du français du XVIIe au XXe siècle », et notamment sur les exercices scolaires ne la fait pas apparaître (Chervel, 2008). Interrogé sur cette question, l'auteur précise, confortant ainsi notre recherche, que "la notion de synthèse de textes paraît totalement absente de la tradition universitaire française jusqu'au XXe siècle ». Pour en expliquer les raisons, lors d'un entretien écrit, il précise :

J'ai essayé, dans mon Histoire de l'enseignement du français, de saisir dans son ensemble la question des manipulations opérées (dans l'enseignement) autour des textes qui sont soumis aux élèves. Toutes impliquent un respect de l'intégrité du texte (et surtout de son contenu), qu'on se prépare à le réciter, à le traduire, à l'expliquer (aux différents sens du terme), voire à le réduire (l'analyse).

Or, la synthèse de textes, se réalisant à partir de différents documents, se prête sans doute moins, à retrouver l'intégrité du contenu de chacun d'eux. Ceci nous amène alors à la questionner comme un « genre » émergeant du rapport de l'étudiant avec les textes écrits. A ce titre, elle demande donc à être située par rapport à d'autres épreuves plus tradition- nelles. Un travail sur cette question a d'ailleurs été mené à l'occasion d'une recherche sur la synthèse (Brassart, 1993). L'auteur repère déjà la diversité des notes de synthèse, son absence dans le secondaire, et cherche à la situer par rapport aux autres genres scolaires devenus classiques dans l'enseignement secondaire du français : avec le résumé de textes, la synthèse partage l'exigence de se référer aux écrits donnés et d'en rendre compte aussi fidèlement que possible ; mais elle se réalise à partir de plusieurs textes et demande une structuration personnelle formulée à partir d'une problématisation. Avec la dissertation, elle partage au contraire la mise en évidence d'une problématique et d'une structure personnelle présentant les différentes dimensions du dossier, mais elle aboutit à l'instruction d'une réponse pratique que la dissertation n'a pas comme enjeu.

Dès lors, en faisant de la « note de synthèse » il y a 40 ans, une condition d'accès à son école, l'ENA a véritablement introduit un nouvel objet. Celui-ci s'est ensuite propagé aux autres établissements de la Fonction Publique recrutant, depuis la deuxième moitié du XXème siècle par voie de concours. Il s'agit d'un objet « transversal » à plusieurs champs, non inscrit dans une discipline, qui devient progressivement l'exercice « emblématique » de l'Enseignement Supérieur (Amorgathe, 1995).

Notre question initiale porte donc sur les écosystèmes de cet objet : " la synthèse de textes peutelle remplir la même fonction dans tous ses habitats?». Ce qui, si nous poursuivons la métaphore écologique s'énonce : «quelles sont les niches de la synthèse de textes? ». Notre enquête, conduite dans l'ensemble de ses 143 habitats, montre, nous l'avons dit, que la synthèse est entrée comme un outil de sélection dans les concours de 117 habitats, puis comme un outil de certification dans les examens. Pour quelles raisons cet exercice est-il apparu dans l'Enseignement Supérieur?

Nous avons ouvert une deuxième question de recherche : "Comment cet objet est-il enseigné ? ", ce qui a abouti à l'identification des écosystèmes (habitats et niches) dans lesquels l'objet « synthèse de textes » vit comme objet d'enseignement. Dans cet article, nous ne nous intéresserons qu'à la première partie du questionnement et nous présenterons donc uniquement les résultats correspondants. 


\section{Approche écologique : "l'habitat " et la « niche "}

Nous retenons de la théorie anthropologique de la transmission des savoirs (Chevallard, 1991) ce qui nous intéresse pour comprendre et expliquer les enjeux liés au rapport institutionnel, à l'habitat et à la niche de la «synthèse de textes au concours »; de ce fait, nous limitons notre cadre au rapport personnel et/ou institutionnel à un objet. Nous mettons en avant l'apport de l'analyse écologique, qui considère les savoirs comme des rapports vivants dans des institutions et marqués par elles, que nous nommons cependant « objets de savoir » puisque pour l'observateur, tout est objet. Ainsi en va-t-il de la synthèse de textes, forme de rapport aux textes. Le rapport institutionnel à la synthèse de textes qui prend, selon les institutions des formes diverses, sera étudié comme variations du rapport aux textes nommé «synthèse » selon les propriétés écologiques de ses habitats, qui correspondent à des niches écologiques diverses si Sy n'y remplit pas les mêmes fonctions, ce que nous allons voir.

Selon cet auteur, deux notions guident l'analyse, l'habitat et la niche d'un objet :

Un savoir donné $S$ se retrouve en divers types d'institutions I qui sont pour lui, en termes d'écologie des savoirs, autant d'habitats différents. Or, à considérer ces habitats, on aperçoit immédiatement que le savoir considéré vient régulièrement y occuper des niches bien distinctes [...] l'habitat, c'est en quelque sorte l'adresse, le lieu de résidence de l'organisme. La niche, ce sont les fonctions que l'organisme y remplit : c'est en quelque façon la profession qu'il y exerce.

L'articulation de ces deux notions permet de définir une méthodologie pour décrire le rapport institutionnel à Sy, soit le R (I ; Sy). Il s'agit d'examiner, dans les lieux où Sy est enjeu didactique, quels sont les autres objets présents, et quel est son rôle dans le système d'objets avec lequel il est en relation. Dès lors, nous rendrons compte du lieu où il est présent, du comment on entre en rapport avec cet objet, ce qui est fait, et ce qui attendu de cet objet (le rapport institutionnel). En étudiant ainsi les conditions d'existence, l'analyse écologique permet de mieux cerner les relations et modes de vie de Sy.

Le travail que nous engageons s'interprète plutôt comme une analyse simple du rapport institutionnel à la synthèse de textes dans différentes institutions où elle apparaît :

- objet : la synthèse de textes Sy. Par convention, on note Syl et Sy2, les objets institutionnels correspondants aux institutions I1, I2.

- institutions : institutions dans lesquelles la synthèse de textes est requise ;

Il : le concours d'admission à l'Ecole Nationale de l'Administration est l'habitat de «Syl»

I2 : le Concours pour le recrutement des Professeurs des Ecoles, en seconde année dans les Instituts Universitaires de Formation des Maîtres est l'habitat de Sy2.

Les deux épreuves Syl et Sy2 sont les deux objets institutionnels que nous choisissons. En effet, à partir des recherches exploratoires, le rapport R (I ; Sy) peut s'organiser dans un continuum allant d'attendus « à caractère fonctionnel dominant $»$ : l'attaché de préfecture doit produire une synthèse à l'image de celles qu'il devra remettre au préfet ; jusqu'à des attendus plutôt « non fonctionnels ». Toutes les « synthèses de textes $»$ recensées relèvent d'un rapport entre ces deux points. Nous choisissons donc deux habitats représentant au mieux ces deux « extrêmes ». En effet, pour Il la synthèse est une compétence professionnelle alors qu'elle est une " compétence non professionnelle» dans l'institution I2.

Par ailleurs, d'autres aspects distinctifs existent :

- Les étudiants qui se présentent ont en général un cursus différent : Droit, Sciences Politiques, pour l'ENA, toutes disciplines pour le CRPE,

- La formation à l'ENA se déroule sur trois années et sur un seul site national, alors que celle de la formation des enseignants se déroule sur une année dans l'un des trente deux sites des Instituts Universitaires de la Formation des Maîtres (IUFM) recensés en 2009.

- Les deux concours proposent des épreuves de synthèses à partir de plusieurs textes mais L'ENA est la première à l'avoir fait (1971). La synthèse de texte du CRPE a été introduite plus récemment (1990). 
- Enfin la nature de l'épreuve, la durée et le nombre de documents sont différents. L'ENA, demande la rédaction, en cinq heures, d'une note permettant de vérifier les qualités d'analyse et de synthèse du candidat ainsi que son aptitude à dégager des solutions appropriées. L'épreuve est constituée d'une question appuyée par un dossier d'une cinquantaine de pages 5 .

L'épreuve proposée pour le CRPE, s'inscrit dans l'épreuve de français. Il est demandé la rédaction en quatre heures, d'une synthèse, permettent de mettre en évidence chez le candidat d'une part, la maîtrise de la langue française et la capacité de compréhension, ainsi que l'aptitude à composer et à rédiger, d'autre part, la connaissance des objectifs, des programmes et des principaux documents d'accompagnement de l'enseignement du français à l'école primaire ainsi qu'une bonne aptitude à les mettre en relation avec la pratique de classe.

L'épreuve de synthèse se réalise à partir d'une question sur trois ou quatre textes relatifs à l'acquisition et à l'enseignement de la langue française.

\begin{tabular}{|c|c|c|}
\hline & Institution $\mathrm{I}_{1}$ & Institution $\mathrm{I}_{2}$ \\
\hline & Concours d'entrée à l'ENA & Concours d'entrée en $2^{\text {nde }}$ année à l'IUFM \\
\hline $\begin{array}{l}\text { Cursus préalable des } \\
\text { étudiants }\end{array}$ & $\begin{array}{l}\text { Faculté de Droit/ Sciences } \\
\text { Politiques/toutes disciplines }\end{array}$ & PEl/Toutes disciplines \\
\hline $\begin{array}{l}\text { Durée de la } \\
\text { formation }\end{array}$ & 3 ans & 1 an \\
\hline Site & Unique & 32 IUFM \\
\hline $\begin{array}{l}\text { Date d'introduction } \\
\text { de l'épreuve }\end{array}$ & Fondatrice : 1971 & $\begin{array}{l}\text { En } 1990 \text {, modification en } 2005 \text {, Projet de modifications } \\
\text { en } 2008\end{array}$ \\
\hline Attendus & $\begin{array}{l}\text { Vérifie des qualités d'analyse et de } \\
\text { synthèse ainsi que l'aptitude à déga- } \\
\text { ger des solutions appropriées. }\end{array}$ & $\begin{array}{l}\text { Vérifie : - la maîtrise de la langue française et la capacité } \\
\text { de compréhension, ainsi que l'aptitude à composer et à } \\
\text { rédiger - la connaissance des objectifs, des programmes et } \\
\text { des principaux documents d'accompagnement de l'ensei- } \\
\text { gnement du français à l'école primaire ainsi qu'une bonne } \\
\text { aptitude à les mettre en relation avec la pratique de classe. }\end{array}$ \\
\hline Durée & 5 heures & 4 heures \\
\hline $\begin{array}{l}\text { Modalité de l'épreuve } \\
\text { de synthèse }\end{array}$ & $\begin{array}{l}\text { Epreuve constituée d'une question } \\
\text { appuyée d'un dossier d'au moins } 50 \\
\text { pages }\end{array}$ & $\begin{array}{l}\text { Epreuve se réalisant à partir d'une question relative à une } \\
\text { partie du dossier : } 3 \text { ou } 4 \text { textes }\end{array}$ \\
\hline Forme & Rendu de quatre à cinq pages & $\begin{array}{l}\text { Le développement attendu est de } 3 \text { à } 4 \text { pages (note de } \\
\text { cadrage, 2005) }\end{array}$ \\
\hline
\end{tabular}

Tableau récapitulatif d'attendus de l'épreuve pour les deux institutions

\section{La synthèse de textes : un habitat de « concours"}

Nous rendons compte de la présence de la synthèse de textes dans deux institutions : ENA $\left(\mathrm{I}_{1}\right)$ et EN $\left(\mathrm{I}_{2}\right)$. Nous faisons d'abord une analyse simple, qui donne une première image des habitats et de l'importance que Sy a ces lieux. Nous repérons sa présence à partir des textes de lois fixant les modalités d'organisation des concours.

\section{Premier habitat de Sy dans l'institution $\mathrm{I}_{1}$}

C'est l'Ecole Nationale d'Administration fondée en 1945, sous l'impulsion de Michel Debré, qui institue la note de synthèse comme un outil de sélection. En effet, la grande réforme du concours de l'ENA, réalisée en 1971, a introduit dans les programmes de ces trois concours, une "épreuve consistant à dégager, en fonction des indications contenues dans le sujet, les éléments essentiels 
d'un dossier et à les mettre en forme d'une manière utilisable », ceci pour permettre le recrutement d'élèves « au profil sociologique et idéologique renouvelé ».
Aujourd'hui, les conditions d'accès sont régies par le décret 2002-50 du 10 janvier 2002. La note de synthèse fait toujours partie des épreuves d'admissibilité. Elle apparaît au même niveau que toutes les autres épreuves.

\begin{tabular}{|c|c|c|c|c|}
\hline $\begin{array}{l}\text { Epreuves du concours d'en- } \\
\text { trée à l'ENA }\end{array}$ & intitulé & Coefficient & Durée & Permet de vérifier s'il y a : \\
\hline \multirow{5}{*}{ Admissibilité } & Droit public & 4 & 5 & \\
\hline & Economie & 4 & 5 & \\
\hline & $\begin{array}{l}\text { Rédaction d'une note } \\
\text { de synthèse }\end{array}$ & 4 & 5 & $\begin{array}{l}\text { - acquisitions de connaissance, } \\
\text { - point d'appui pour l'élaboration d'un plan } \\
\text { d'action } \\
\text {-orientation pour la prise de décision en vue } \\
\text { d'une action }\end{array}$ \\
\hline & $\begin{array}{l}\text { Evolution générale } \\
\text { politique }\end{array}$ & 4 & 5 & \\
\hline & Langue & 2 & & \\
\hline
\end{tabular}

Epreuves du concours d'entrée à l'Ecole Nationale de l'Administration

Si l'on compare ces différentes épreuves d'admissibilité, la dernière épreuve (celle de la langue) paraît relative, l'attente semble être moindre. Concernant la synthèse par contre, l'Ecole accorde à celle-ci une place importante, compte tenu de son coefficient élevé et de sa stabilité dans le temps. Sy ${ }_{1}$ apparaît dans $\mathrm{I}_{1}$ comme « objet de concours ».

\section{Second habitat de Sy $y_{2}$ dans l'institution $I_{2}$}

L'épreuve de synthèse de textes dans le concours de recrutement des professeurs des Ecoles apparaît tardivement, à l'occasion d'une réforme du statut des enseignants du primaire (décret du $1^{\text {er }}$ aout 1990). Cette épreuve coincide avec la mise en place des IUFM et d'une dimension universitaire de la formation. Elle est donc requise dans les épreuves d'admissibilité depuis 1990. Sy ${ }_{2}$ est, dès son origine, travaillé dans la discipline du français. Il fait l'objet de modifications en 2005 et reste objet de discussion depuis 2008 dans le cadre de la "mastérisation » des concours de recrutement des enseignants ${ }^{6}$. On remarque plusieurs appellations de Sy2 : de 1990 à 2005, les textes de références, les sujets, les rapports de jury écrivent : synthèse de textes et de documents (1990), synthèse de documents (2000), synthèse de textes (2001), note de synthèse (2003). De 2005 à
2009 , les termes utilisés deviennent : synthèse, question de synthèse (2006), travail de synthèse (2008).

De manière générale, il y a toujours eu deux grandes épreuves d'admissibilité dans ce concours : une épreuve de français qui a, pour objectif, avant 2005 « de mettre en évidence la capacité de compréhension, l'aptitude à comparer et à rédiger, ainsi que la maîtrise de la langue du candidat. L'épreuve sert aussi à vérifier la connaissance des objectifs et des programmes de l'enseignement de la langue française à l'école primaire, ainsi qu'une bonne appréciation des approches didactiques et des démarches pédagogiques ${ }^{7}$ » et une épreuve de mathématiques. L'épreuve de français comportait deux volets. Dans le premier, le candidat faisait la synthèse de textes des documents relatifs à l'acquisition et à l'enseignement de la langue française et repérait les erreurs dans la production écrite d'un élève ; dans le second, il analysait et critiquait des documents pédagogiques relatifs à cet enseignement à l'école primaire.

Depuis le 26 mai 2005 (texte actuel de référence), l'épreuve se présente comme suit : » à partir d'un dossier composé de textes et de documents relatifs à l'acquisition et à l'enseignement de la langue française, le candidat : - effectue une synthèse à partir 
d'une question relative au dossier ; - traite un thème ayant trait à la grammaire ; - répond à une question complémentaire sur la mise en situation d'enseignement d'une ou plusieurs notions abordées dans le $\operatorname{dossier}^{8} »$.
La durée totale de l'épreuve est de 4 heures et d'un coefficient de 3. L'épreuve est notée sur 20 points : 8 points sont attribués à la synthèse, 4 points au thème relatif à la grammaire et 8 points à la question complémentaire.

\begin{tabular}{|c|c|c|c|c|c|}
\hline Epreuves du CRPE, & intitulé & & Coefficient & durée & Permet de vérifier \\
\hline \multirow[t]{2}{*}{ A d missibilité } & $\begin{array}{l}\text { Epreuve écrite de } \\
\text { français }\end{array}$ & $\begin{array}{l}\text { - Synthèse à } \\
\text { partir d'une } \\
\text { q u e s t i o n } \\
\text { (8pts) } \\
\text {-Grammaire } \\
\text { (4pts) } \\
\text {-Question c } \\
\text { (8pts) }\end{array}$ & 4 & 5 & $\begin{array}{l}\text {-capacité de compréhension, aptitude à } \\
\text { comparer et à rédiger } \\
\text {-maîtrise de la langue du candidat } \\
\text {-vérifier la connaissance des objectifs et } \\
\text { des programmes de l'enseignement de la } \\
\text { langue française à l'école primaire } \\
\text {-bonne appréciation des approches didac- } \\
\text { tiques et des démarches pédagogiques. }\end{array}$ \\
\hline & Mathématiques & & 4 & 5 & \\
\hline
\end{tabular}

$\mathrm{Sy}_{2}$, noté sur huit points, correspond à la première des trois parties du volet « épreuve écrite de Français ».

Ce premier niveau d'analyse permet de montrer que le concours commande les habitats de Sy, concours d'admission dans une Ecole pour $\mathrm{Sy}_{1}$, concours de recrutement à une profession via une formation mise en place par un institut universitaire pour $\mathrm{Sy}_{2}$.

Par ailleurs, la place de Sy dans les deux institutions Il et I2 est différente. En effet, dans I1, Syl est présent en personne, alors qu'il partage son habitat et occupe un plus petit volume dans $\mathrm{I} 2$. $\mathrm{No}^{-} \neq$ supposerons, par méthode, que Syl =RIl (textes) $\neq$ Sy2 =RI2 (textes) et pour cela nous en rechercherons les fonctions.

\section{Les fonctions de la « synthèse de textes"}

Pour étudier la vie de Sy, nous choisissons de regarder les textes de lois, les notes de service, ainsi que les rapports de jury. Nos questions sont les suivantes: qu'est ce que l'objet $\mathrm{Sy}_{1}$ ? Qu'est ce que l'objet $\mathrm{Sy}_{2}$ ? Dans quelles conditions existent-ils ?
Quelles relations entretiennent-ils dans leur institution respective $\left(I_{1}-I_{2}\right)$ ?

\section{Présentation des objets $S y_{1}$ et $S y_{2}$ par leur institution}

Pour Syl, les intitulés prennent souvent des formes favorisant la mise en situation du candidat : «vous êtes Conseiller technique du Ministre ${ }^{9}$ ", «Conseiller des Affaires Etrangères à la Direction des Affaires Juridiques du Ministère ${ }^{10} »$ ou encore, « vous travaillez pour votre Directeur de cabinet pour le Secrétaire Général du Ministère ${ }^{11}$ ". Les questions peuvent être relatives à une situation particulière, par exemple : «plan social de l'entreprise Martin sur des coopérations renforcées ${ }^{12} »$. Il peut être demandé au candidat d'apprécier « la pertinence des solutions et de formuler « toute proposition utile ${ }^{13} »$. Il est également appelé à " évaluer le dispositif existant", ou encore à " proposer des orientations pour une éventuelle modification ${ }^{14}$ ».

Les attentes de cette note, présentée en même temps qu'un dossier de dix sept documents d'une totalité de quarante cinq pages ${ }^{15}$ s'expriment souvent dans l'intitulé : «Vous êtes chargé de mission auprès d'un Recteur d'Académie. Vous sont demandés les grandes 
lignes d'un projet de plan de lutte contre les violences en milieu scolaire. Votre note doit comporter :

- une présentation générale du plan proposé, avec la détermination de priorités et d'un calendrier de mise en œuvre.

- une fiche technique qui permette aux établissements et écoles de suivre et d'évaluer les mesures qu'ils mettent en œuvre ${ }^{16}$ ».

Dans tous les cas, la note est vouée à une utilisation pratique et immédiate, d'une position asymétrique, d'un « collaborateur » à un « décideur». Elle doit être pour ce dernier à la fois, un résumé, une mise au point, un aide-mémoire, un canevas en vue d'une intervention à accomplir. L'aptitude au raisonnement (juridique, économique, social, international) doit sous-tendre l'analyse du dossier, et la note est avant tout l'expression ciblée d'une analyse, de propositions, et traduit l'aboutissement de la réflexion ${ }^{17}$.

Avant 2005, Sy ${ }_{2}$ comptant pour 8 points sur l'ensemble de l'épreuve, conduit à des dossiers conçus pour être traités en une heure et demie environ ${ }^{18}$ ne dépassant pas deux mille mots ${ }^{19}$, avec des choix de sujets "sans visée trop technique ${ }^{20}$ ". L'intitulé du sujet est de forme comparable d'année en année quelle que soit l'Académie en France. Le candidat est invité à rédiger ${ }^{21}$, présenter ${ }^{22}$, établir $^{23}$, à partir de trois ou quatre textes, en trois pages manuscrites, une note de synthèse ${ }^{24}$. Parfois des précisions soulignent les attentes prescrites dans les recommandations officielles : « sans porter d'appréciation personnelle ${ }^{25}$ », ou « en vous interdisant tout avis personnel $^{26}{ }$ vous rédigerez une synthèse d'environ trois pages en vous appuyant sur l'ensemble des éléments du dossier (textes et documents) ». La note a pour finalité contrairement à $\mathrm{Sy}_{1}$, de permettre au jury d'apprécier chez le candidat « sa capacité à lire et à comprendre les textes ainsi que son aptitude à saisir l'essentiel d'un problème et à l'exposer avec justesse dans une langue correcte ${ }^{27}$ ", avec une évaluation " s'intéressant au tri des idées qu'a su opérer le candidat, à son aptitude à distinguer l'essentiel de l'accessoire, à sa façon d'opérer des raccourcis et d'user d'une formulation dense et concise ${ }^{28} »$.

Depuis 2005, les IUFM ont continué à travailler dans cet esprit de confrontation de textes portant sur le même objet, mais en formulant désormais une ques- tion dans l'intitulé du sujet. La nouveauté de l'épreuve, suite à l'introduction de cette modification, oriente le lecteur vers la problématique sous jacente aux textes.

Les intitulés prennent alors des formes permettant de présenter les ressemblances, les divergences sur un problème de grammaire, d'orthographe, de lecture, d'écriture etc... Ils se présentent en général sous cette forme : "Vous rédigerez une synthèse en vous attachant à préciser les conditions favorables à l'acquisition du vocabulaire chez les élèves ${ }^{29}$ ", ou « les enjeux de l'enseignement de l'oral dans l'articulation entre langue de la maison et langue de l'école ${ }^{30}$ ", « vous préciserez quelles sont les spécificités de la lecture documentaire à l'école primaire ${ }^{31}$ », « vous préciserez en quoi la lecture de textes de littérature de jeunesse facilite l'organisation des différents aspects de l'enseignement de la productio de textes au cycle $3 »$.

Ainsi, les intitulés de l'épreuve, le support de la note, la consistance du dossier et la justification de sa programmation sont propres à chaque institution. Pour $\mathrm{Sy}_{1}$ il y a une certaine constance dans le R $\left(\mathrm{I}_{1}\right.$; $\mathrm{Sy}_{1}$ ) alors que ce rapport se modifie dans la durée pour $\mathrm{Sy}_{2}$. Cela nous amène à prendre en compte le contexte dans lequel l'objet est pris et l'attente qui porte sur lui.

\section{Le contexte de $S y_{1}$ et $S y_{2}$}

Sy ${ }_{1}$ ne sert pas seulement de soubassement pour mesurer des acquisitions de connaissance : "Les candidats doivent montrer dans leur synthèse des connaissances relatives aux étapes de la construction européenne, aux institutions, aux processus décisionnels [...] aux finances de l'Union Européenne [...] ainsi que tout ce qui va concerner les questions sociales allant du travail salarié, aux politiques de l'emploi, à la protection sociale et à la solidarité »; il est également point d'appui pour l'élaboration d'un plan d'action et sert enfin à orienter la prise de décision pour une action.

L'objet synthèse apparaît d'emblée dans une interrelation étroite avec l'objet " plan d'action », dans la mesure où Syl sert de repère pour élaborer celui-ci. On comprend mieux son contexte, dès lors que l'on s'intéresse aux attentes qui sont faites. Le rapport de jury du concours de l'année 2000 écrit : « une 
question est posée sur ce dossier qui doit permettre de juger la capacité de compréhension, d'analyse de synthèse, de classement des idées et de rédaction des candidats [...] c'est une épreuve technique d'un collaborateur $»$. Cet extrait du rapport indique que la note de synthèse permet de vérifier que le candidat est bien dans un rapport collaborateur/note/décideur. On sélectionne un futur candidat capable de proposer un écrit utilisable pour autrui. Mais ce changement déclaré du « candidat, copie, correcteur » à celui de " collaborateur, note, décideur » est-il aussi simple et évident qu'il y paraît ? Ou suppose-t-il, au contraire, un changement profond de l'ordre du rapport que l'étudiant entretient habituellement avec une épreuve?

Avant les modifications de 2005, et contrairement à $S y_{1}$, les liens entre $S y_{2}$ et les autres parties de l'épreuve de français sont à rechercher. $S y_{2}$ ne semble ni servir à l'épreuve consistant à « repérer les erreurs dans la production écrites d'un élève », ni à celle liée à « l'analyse critique des documents pédagogiques relatifs à l'enseignement $»$. On note cependant, que les recommandations faites aux concepteurs de sujets de même que les critères d'évaluation donnés aux correcteurs ont toujours cherché à valoriser la mise en relation des deux parties. Il était demandé de « mettre en relation le second volet avec le premier chaque fois que cela était possible » (rapport de jury 2001-2003). Jusqu'en 2005, les relations entre les parties de l'épreuve de français ne sont pas données mais relève plutôt de l'implicite du concepteur de l'épreuve - à charge pour l'étudiant d'imaginer une reconstruction hypothétique.

À partir de 2005, la note de cadrage stipule pour les concepteurs que le « dossier » de support à l'épreuve doit être constitué de textes (3 ou 4) et de documents relatifs à l'enseignement, et qu'il doit présenter « une unité thématique ». Pour les correcteurs, il est précisé que la question de synthèse peut «porter sur tout ou partie du dossier ».

Ainsi, les trois parties du dossier sont désormais en étroite relation et supposent donc une approche globale. Des rapports de jury conseillent d'ailleurs aux candidats de prendre connaissance de l'ensemble du dossier avant de traiter la partie «synthèse " (rapport de jury 2008). L'épreuve semble ainsi organisée autour d'une rhétorique de confrontation et de problématisa- tion pour l'explicitation des enjeux de l'enseignement du français, plutôt qu'autour d'une restitution de savoirs. Mais pour quelle manipulation?

Nous avons distingué les contextes de la présence de $S y_{1}$ et $S y_{2}$, en caractérisant les réseaux de relations présents pour $S y_{1}$ et $S y_{2}$ et en repérant des attentes dans les institutions $\mathrm{I}_{1}$ et $\mathrm{I}_{2}$. Ainsi, même si nous remarquons que les institutions accordent un crédit important à l'épreuve de synthèse, c'est un objet différent. Chacune a donc une connaissance précise de ce qui est attendu, sans connaître le rapport différent que l'objet Sy entretient avec chaque institution. Pour poursuivre notre analyse, nous allons regarder la manière dont $S y_{1}$ et $S y_{2}$ sont manipulés dans la pratique professionnelle.

\section{La manipulation de $S y_{1}$ et $S y_{2}$ dans la pratique}

Les agents de l'ENA utilisent $\mathrm{Sy}_{1}$ dans leurs activités quotidiennes. C'est un outil de communication. Réaliser des notes à l'attention d'un supérieur est un geste de cette pratique. Cet « attendu fonctionnel » est utilisé dans une relation asymétrique : du collaborateur (qui produit la note), vers un décideur (qui la commande et l'évalue dans un rapport hiérarchique). La fonction de la synthèse est donc de faciliter une communication à l'articulation entre un collaborateur et un décideur : celui qui la réalise n'est pas non plus celui qui décide de l'action. En 1990, dans le cadre de l'ouverture de l'ENA à la société civile (Anciens élus locaux, syndicalistes, responsables associatifs, acteurs du privé), se développent les concours de troisième voie. Considérés comme peu affiliés à l'institution, ces élèves vont durant les vingt-sept mois de scolarité réaliser notes de synthèse sur notes techniques et être affectés dans quatre lieux différents. Cet exemple illustre une manipulation de $S y_{1}$, tout autant orienté sur le contrôle de ses agents que sur un mode d'affiliation dans une relation d'acculturation. Par ailleurs, la reconnaissance de ce geste distinctif des hauts fonctionnaires d'état, pourrait aussi avoir fait l'objet d'un début de transposition dans le cadre des épreuves de recrutement pour les concours externes, d'abord de L'ENA puis des concours de catégorie A. L'Ecole chercherait donc à déceler les aptitudes des candidats à ce geste professionnel. Ce serait donc une épreuve qui désignerait, par avance, les codes écrits d'affiliation à l'institution professionnelle. 
Les agents de $\mathrm{I}_{2}$ utilisent $\mathrm{Sy}_{2}$ de manière très différente. Dans les programmes et instructions issus des bulletins officiels, $S_{2}{ }_{2}$ 'est pas, ou très peu, directement requis. Si la fonction originaire de $\mathrm{Sy}_{1}$ se découvre comme un moyen de communication professionnelle entre différents agents en position asymétrique, cette fonction disparaît dans l'habitat de l'Education Nationale où le Professeur n'a pas à communiquer, ni dans le cadre d'une relation hiérarchique, ni dans le cadre de sa relation conjointe avec l'élève. D'où cette première observation : la fonction de $\mathrm{Sy}_{2}$ n'est donc plus la même que celle occupée dans sa niche initiale. Seconde observation, le rapport de $\mathrm{Sy}_{2}$ se construit entre les Instituts Universitaires de Formation des Maîtres et les candidats se destinant à devenir Professeurs des Ecoles.

Il y a donc, deux positions qui se dégagent $\mathrm{e}=\mathrm{la}$ positon de l'élève et $\mathrm{P}=$ la positon du Professeur. Le rapport, pour les sujets de l'institution, occupant la position d'élève, à l'objet $S_{2}{ }_{2}$ pourrait ne pas exister puisque ne faisant pas partie des savoirs à enseigner ; d'où un rapport de l'élève à l'objet qui, dans certain cas peut être vide : $\mathrm{R}_{\mathrm{I}}\left(\mathrm{e} ; \mathrm{Sy}_{2}\right)=\varnothing$. Par contre, le rapport pour les sujets de l'institution, occupant la position de Professeur existe. Il prend son origine dans le moment de la formation conduisant au CRPE. On peut alors s'interroger sur la relation de ce rapport $\mathrm{R}_{\mathrm{I}}\left(\mathrm{P} ; \mathrm{Sy}_{2}\right)$.

La fonction de Sy2 est de dépasser la visée des épreuves de " restitution de savoirs » en favorisant une confrontation de textes proposant des perspectives différentes sur le même objet. Les bonnes copies sont celles qui précisément ont " manifesté cette compréhension d'ensemble et ont su utiliser tous les éléments du dossier dans un éclairage mutuel » (jury 2008). Or, le rapport aux textes demandé à l'étudiant semble en rupture avec le modèle traditionnel : » respect, intégrité d'un seul texte, qu'on se prépare à réciter, à traduire, à expliquer, voire à réduire ${ }^{35} \%$.

En quoi alors, le contexte de la synthèse, en prenant l'activité de l'étudiant, influe-t-il, même d'une manière inconsciente, sur son rapport aux textes et donc aussi sur sa posture?

A priori, l'étudiant aborde moins la lecture des textes du dossier sous la forme de : «Qu'est-ce que chaque texte veut dire? ». Mais plutôt à travers la question de : "Qu'est-ce que ce texte veut dire par rapport aux autres ? ». Il s'agit donc plutôt d'une lecture répondant potentiellement à un système de relation : ce n'est plus une lecture fermée mais une lecture visant a priori la construction de passerelles permettant de construire un " rapport à ...». L'étudiant cherche d'abord à répondre à cette question : «En quoi peut-on mettre les textes en dialogue? » Ce n'est que dans un second temps qu'il s'intéresse à l'élaboration d'une codification : «Ce sur quoi on veut mettre ces textes en dialogue correspond-il vraiment à leur contenu ?». Ce questionnement étant contextualisé, interviendrait aussi le fait qu'il est très difficile de les lire en faisant abstraction de tout l'intertexte potentiel, et donc de toute la vulgate communément admise (chacun des textes fait allusion à un contexte d'apprentissage comme la lecture, l'écriture, la grammaire, etc....).

D'un rapport aux textes, où chacun d'eux se réduit à des éléments simples, succède un rapport aux textes fondé sur un principe de simultanéité, d'interaction (Kant, 1787). À un modèle d'objectivité issu d'une certaine approche du réductionnisme, succéderait ainsi, en l'incluant, un modèle prenant en charge le caractère interactif de la subjectivité. Il s'agirait alors, d'un système structurel qui « s'élabore au croisement de surfaces textuelles » (Kristeva, 1978). Cette hypothèse se révèle radicalement différente de l'approche traditionnelle des systèmes d'enseignement, ce qui confère, par ailleurs à $S_{2}$, un statut épistémologique particulier. Mais le système de formation des Professeurs des Ecoles, tel qu'il s'est construit depuis 1990, permet-il à l'étudiant ce rapport aux textes?

\section{Conclusion}

L'approche écologique a permis de décrire la niche de la « synthèse de textes » dans deux institutions [ENA - E.N], choisies comme habitats les plus contrastés parmi les 117 repérés dans l'Enseignement Supérieur. C'est seulement dans le cadre des concours de recrutement de la fonction publique que la synthèse devient « objet d'enseignement » et plus précisément un « objet de concours ». Par ailleurs, en remontant du concours à sa fonction professionnelle, nous montrons que si Sy s'inscrit dans un 
usage professionnel pour les agents de l'ENA, cela ne s'observe pas si facilement pour les agents de l'Education Nationale. Il y a donc un détournement de sa fonction initiale : la synthèse n'occupe plus la même fonction que celle occupée dans sa niche originelle. Trois questions s'ouvrent :

- la première question est d'ordre méthodologique. Pouvons-nous situer la synthèse exclusivement dans une ligne de partage entre une niche «fonctionnelle » et/ou « abstraite »? Dans sa fonction de sélection par la voie des concours de la Fonction Publique d'Etat, nous n'avons pas trouvé d'autres attendus. Par contre sa migration depuis les années 1971, contamine désormais les concours de catégorie B, mais aussi les institutions d'enseignement post baccalauréat, de formation, publiques ou privées (Ecoles de commerce...). Ce phénomène s'étend même dans le secondaire avec une synthèse demandée dans le cadre du baccalauréat francoallemand. Ces institutions utilisent désormais cet objet comme un élément de concours ou de certification. Mais, les programmations sont d'autant plus précaires que les attendus ne reposent pas sur un aspect fonctionnel établi. Dans ce cas, on peut alors avoir des synthèses dont la consigne s'apparente à un questionnaire à choix multiple (QCM) prenant appui sur les cours réalisés dans le cursus d'enseignement (Faculté de médecine), Ainsi, Sy commence à avoir d'autres fonctions que celles décrites dans le continuum « fonctionnel » « abstrait».

- La seconde question concerne son habitat principal. L'analyse réalisée met au jour le « concours » comme la raison première du développement de la « synthèse écrite ». C'est finalement lui qui commande aux autres habitats institutionnels. Dès lors, nous pouvons questionner cet objet dans l'écosystème où il se généralise : « les concours républicains de recrutement ». Car, à l'interroger, on lui découvre une dimension étroitement imbriquée au système du «vivre ensemble » à la française et par là même spécifique aux pays francophones. Le recours au concours pour les fonctionnaires est prévu dans la loi du 19 octobre 1946. Il s'agit de permettre un mode de recrutement démocratique. Il vise à assurer l'égalité des chances entre les candidats en permettant une qualité de recrutement fondé sur la seule compétition. «L'épreuve de synthèse » (1971) est alors conçue comme un outil de sélection permettant de réaliser cette discrimination souhaitée mais égalitaire. Mais sur quoi se fonde-t-elle tant les écologies restent locales?

C'est en comparant l'enseignement proposé à l'occasion de sa préparation aux concours par rapport aux systèmes d'enseignement bureaucratique ou aristocratique que l'on pourrait travailler un premier niveau de réponse. En effet, selon la distinction opérée par M.Verret (1975), les systèmes modernes d'enseignement, sont dit efficaces dans un système démocratique, s'ils désignent explicitement le savoir à étudier par un plan d'études ou de programme. Cette efficacité est liée à « l'introduction d'une rationalisation des savoirs " (Mercier, 2004). Ce système est aujourd'hui préféré à celui plus ancien appelé « système aristocratique » qui consiste en un enseignement fondé sur l'apprentissage par frayage et choix électif réciproque du maître et de l'élève.

Dans cette option, il s'agit donc de déterminer si une didactique de la « synthèse de textes " peut être explicitement désignée par un plan d'étude ou si elle relève plutôt d'un système par frayage. En raison du peu de référence dont elle dépend (déplacement de sa fonction et peu de savantisation de l'objet), on peut supposer que l'appropriation de ses formes concrètes s'apparente pour partie, à un « rite d'affiliation aux codes écrits de l'institution»(Pérez, 2008).

- La dernière question concerne l'histoire transpositive de Sy. En effet, si sa programmation se comprend à l'ENA où il est un « geste professionnel » des fonctionnaires de l'Administration, en revanche, il reste à interroger à propos des agents de l'Education Nationale, où il perd cet aspect «fonctionnel ». Que pourrait-on alors dire de cette migration? Originairement, la note de synthèse est une pratique sociale des agents de l'ENA, devenue ensuite un exercice de recrutement. C'est par un phénomène de généralisation des concours que va se développer l'usage de ce terme spécifique. Le besoin de synthèse des institutions professionnelles (Administration, Magistrature...) amènent une programmation de l'exercice dans les institutions de recrutement (par les programmes), pour finalement se diffuser aux institutions didactiques (nécessité de préparer les étudiants). Ce serait un phénomène issu d'une transposition didactique par « le haut» (Poucet, 2004) et véhiculé par les concours de recrutement. Ce « geste professionnel » des fonctionnaires de l'Administra- 
tion, s'est ainsi trouvé progressivement dévolu à l'institution didactique "Université » par ce processus : «Attendus (gestes) » professionnels $\rightarrow$ Institutions de formation $\rightarrow$ Institutions de recrutement $\rightarrow$ Concours $\leftarrow$ Institution didactique « Université » (préparation).

Mais cette épreuve, par la suite, a même été retenue dans les cas où il n'y avait pas de justification professionnelle. Depuis 1990, $\mathrm{Sy}_{2}$ s'est construit en valorisant une rhétorique de confrontation et de problématisation autour de l'explicitation des enjeux de l'enseignement du français plutôt qu'une valorisation d'une restitution de savoirs. Cette approche peut se mettre en lien avec l'évolution de la profession qui considère aujourd'hui que « l'enseignant transmet non seulement des objets culturels mais aussi son propre rapport à la culture et aux savoirs de sa discipline » (Rochex, 1998). Pour l'enseignant donc, transmettre les clés de la structure scolaire signifie " témoigner, à travers son activité pratique et discursive, de la signification qu'il lui attribue ». Il s'agit de prendre en compte dans la formation autant son rapport aux contenus que ces contenus stricts. La posture de l'enseignant ne peut plus donc être construite sur un rapport aux textes allant du simple au complexe. Il s'agit plutôt de créer les conditions contextuelles de l'affrontement entre les « concepts » et représentations expérientielles de l'étudiant et les savoirs académiques (savoirs savants transposés). Le « savoir objectif » migre ainsi vers un « rapport à... » incluant dans son système "l'objet de savoir ». Ce « rapport à... » prend sens dans sa structure et ne peut être saisi que dans sa circulation entre l'énonciateur et l'énonciataire du discours. Des travaux s'inscrivant dans ce champ ${ }^{36}$, conduisent à s'intéresser « au caractère interactif de la subjectivité » (Fontanille, 1989); d'où un rapport aux textes fondé dans une confrontation saisie par une subjectivité « conventionalisée » au sens de E. Nonnon (Nonnon, 2008), ou encore par une » lecture contractuelle entre coutume et émergence » (Mercier, 2009).

Former les enseignants signifie alors mettre en place les conditions d'une dialectique entre son activité et sa subjectivité. La lecture intertextuelle sollicitée par $\mathrm{Sy}_{2}$ introduirait certainement l'étudiant dans des champs de débats nouveaux pour lui. Face à ces perspectives, on ne peut que constater le porte-àfaux de l'introduction de la professionnalisation. Les épreuves du concours des enseignants du premier degré, telles qu'on les connaît aujourd'hui s'originent dans une réforme du statut, mêlant promotion (fonctionnaire de catégorie A), passage du «métier» à la « profession » (adaptation à des situations complexes, réflexivité), universitarisation de la formation (IUFM) mixant sur deux ans début de formation professionnelle et préparation d'un concours. La logique de $\mathrm{Sy}_{2}$ fait apparaître la formation dispensée en France pour construire ce type de capacité professionnelle comme un « hybride, sans cohérence interne, de logique universitaire et de professionnalisation (Bernié § Pérez, 2009). Le contexte français rend urgente la réflexion sur le haut niveau disciplinaire requis. En l'état, ce critère seul ne peut pas répondre à la question de l'adaptation à des situations complexes d'un praticien réflexif : « on ne peut pas transmettre la culture comme on transmet un témoin dans une course de relais » (Rochex, 1998).

Ce phénomène transpositif du passage de la synthèse a des fonctions différentes nécessiterait alors une recherche spécifique avec comme question : qu'est-ce que les institutions essaient de transporter comme valeurs en transposant ainsi ces objets et en les détournant de leur fonction initiale ? Nous en avons esquissé les grands axes pour $\mathrm{Sy}_{2}$. Le seul point commun entre tous ces exercices, est que la réalisation d'une note de synthèse se fait toujours en réponse à une question. Cette demande porte essentiellement sur la réalisation d'une enquête dans un monde limité (par le volume du dossier et le temps de l'épreuve). L'étude dans cet article, de deux objets contrastés, issus des concours de recrutement met au jour cette caractéristique. Sans cette attente, il n'y aurait pas de demande, ni même d'existence de l'objet. Dès lors, sortant de la logique de restitution d'une unité simple pour solliciter la mise en perspective des éléments d'un tout, la synthèse s'originerait dans le même contexte ayant rendu possible la vogue de la notion de « compétence ». « Connaître » reviendrait à retrouver les savoirs pertinents par rapport à la question, les montrer et les réorganiser pour y répondre.

D'autres exercices, comme celui de " Restituer de manière Organisée des Connaissances » (ROC) en mathématiques, rendre compte d'une enquête par une affiche dans le champ des " travaux personnels encadrés ", s'inscrivent dans ce contexte. Il y a donc bien une diffusion de cette attente, au-delà de l'enseignement supérieur. 
Ainsi, une « écologie sociale » se dégage en dehors, ponctuellement, de « l'écologie locale». Elle définit le savoir comme étant une « compétence pour répondre à des questions à partir d'un corpus donné ». Or, ce qui a produit les disciplines, et donc l'enseignement dans un système didactique bureaucratique - l'école d'aujourd'hui -, est fondé sur la possibilité de pouvoir déclarer publiquement, les procédures, les contenus, et une évaluation. Mais, en légitimant les « compétences » dans le champ disciplinaire, ce n'est plus le cas. Comment Dès lors produire une publicité et une évaluation pour les objets ainsi introduits ? 


\section{NOTES}

1. L'Université entendue dans le sens moderne de « ensemble de l'Enseignement Supérieur » (Chervel, 1988).

2. Conditions fixées par le décret 72-355 du 4 mai 1972 et par l'arrêté du 5 mars 1973. L'ENA a été suivie par l'Ecole Nationale de la Magistrature qui dans son règlement du premier, deuxième et troisième concours d'accès, prévoit au nombre des épreuves d'admissibilité, une note de synthèse.

3. Originairement, nous avons délimité notre champ de recherche, par l'Université définie comme ci-dessus. Il s'agissait d'aller regarder dans tous les établissements, c'est à dire dans les Universités, les Ecoles publiques ou privées placées sous la tutelle de différents ministères, qui assurent un enseignement supérieur à finalité professionnelle, les classes préparatoires aux grandes écoles, les sections de techniciens supérieurs et les instituts universitaires de formation des maîtres, où la synthèse de textes pouvait être présente afin de mesurer dans un premier temps l'ampleur du phénomène. Cependant l'éclatement de cet enseignement supérieur nous obliger à une approche originale pour les retrouver, et nous avons choisi les textes législatifs, seuls documents communs à chacun des établissements. (Tableau récapitulatif de tous les textes législatifs consultable sur l'annexel de la thèse de J.-M. Pérez 2007). Une fois les documents identifiés et les épreuves retrouvées (recherche par mot-clef : "épreuve de synthèse de textes ", " synthèse de textes ", " note de synthèse ») nous avons pu les comptabiliser et repérer le niveau d'enseignement où elles se trouvaient (selon le système «Licence, Master, Doctorat»), tout ceci afin de répondre à une seule question: Quelle est l'ampleur de diffusion de cet exercice dans l'enseignement supérieur ? - Nous avons ainsi recensé cent quarante trois épreuves de synthèse de textes. Notre article s'est recentré sur le «bloc » le plus consistant tant par l'origine, l'historique, la régularité et l'inscription. Reste qu'il peut être décliné comme suit : - 119 épreuves sont programmées dans un cadre organisé pour le recrutement de la Fonction publique. En effet, le concours en France est le moyen principal pour recruter les futurs fonctionnaires Le rapport de ces concours avec l'Enseignement Supérieur existe bien, en ce sens où, une fois admis, les lauréats rejoignent une école créée par l'Etat, d'une durée moyenne d'un an, pouvant aller jusqu'à trois ans. C'est dans ce cadre là par exemple que se retrouve l'épreuve de synthèse au Concours externe de Chef de travaux d'art, de conservateur des Bibliothèques, d'Officier de la Gendarmerie Nationale, d'Ingénieur d'études sanitaires, ou encore celle du Recrutement des Professeurs des Ecoles (C.R.P.E). Ce sont ainsi autant d'institutions distinctes qui organisent ces exercices de synthèses. Les 24 épreuves restantes concernent 245000 étudiants en sections techniques supérieures, 72000 étudiants en classes préparatoires aux Grandes Écoles et 111 OOO étudiants en écoles paramédicales. Au final, pour chaque année universitaire ce sont au moins 500000 étudiants qui sont directement concernés par une épreuve de synthèse de textes.

4. Verret (1975) distinction de l'enseignement bureaucratique et de l'enseignement aristocratique

5. Décret $\mathrm{n}^{\circ}$ 2002-50 du 10 janvier 2002 relatif aux conditions d'accès et aux régimes de formation de l'Ecole Nationale d'Administration.

6. Note de service 94-271 du 16 novembre 1994 ; arrêté du 10 mai 2005 ; et discussion dans le projet dit de « Mastérisation » des concours de recrutement des enseignants.

7. Arrêté du 29 avril 2002 modifiant l'arrêté du 18 octobre 1991 fixant les modalités d'organisation du concours externe de recrutement de professeurs des écoles.

8. B.O n²1 du 26 mai 2005.

9. Concours d'entrée à l'Ecole Nationale de l'Administration 2000

10. Concours d'entrée à l'Ecole Nationale de l'Administration 1999

11. Concours d'entrée à l'Ecole Nationale de l'Administration 2001

12. Concours d'entrée à l'Ecole Nationale de l'Administration 2001

13. Concours d'entrée à l'Ecole Nationale de l'Administration 2001

14. Concours d'entrée à l'Ecole Nationale de l'Administration 1999

15. Ecole Nationale de l'Administration, sujet et meilleures copies du concours 2001 p.319 
16. Dossier de synthèse E.N.A, session 2001

17. Concours d'entrée à l'Ecole Nationale de l'Administration 2001

18. Note de service 94-271 P.2

19. Note de service $94-271$ P.3

20. Note de service 94-271 P.3

21. Sujet 2001 Académie d'Aix Marseille

22. Sujet 1996 Académie de Reims

23. Sujet 1996 Académie de Lyon

24. Dossier de synthèse C.R.P.E, session 2000

25. Sujet de 1994 et sujet de 1997 Aix (sans appréciation personnelle) ; sujet de 1996 Besançon (rappel : la note de synthèse n'est pas un résumé linéaire)

26. Sujet de 2003, Groupement interacadémique II

27. Note de service 94-271 P.2

28. Note de service 94-271 P.2

29. Sujet 2009 Orléans-Tours FRA-09-PG1

30. Sujet 2009 FRA-09-PG2

31. Sujet 2008 Lille FRA-08-PG5

32. Sujet 2006 Lille FRA-06-PG6

33. Arrêté du 13 octobre 1999 fixant les programmes dans les épreuves de concours d'entrée à l'école Nationale de l'Administration

34. Voir page 3 : A. Chervel

35. Kant 1738 : « toutes les substances, en tant qu'elles peuvent être perçues comme simultanées dans l'espace, sont dans une action réciproque universelle » (p. 219)

36. Sous l'impulsion des travaux de Bakhtine (1978)

\section{RÉFÉRENCES}

Amorgathe, D. (1995). La synthèse de documents. Paris: Dunod.

Bernié, J.P, Pérez, J.M. (2009). Aide personnalisée et clés de la culture scolaire : une interpellation de certains principes en didactique et formation. Communication acceptée au Congrès International de Didactiques. L'activité de l'enseignant: Intervention, Innovation, Recherche. Gérone. Espagne. Février.

Brassart, D. G. (1993). Remarques sur un exercice de lecture-écriture : la note de synthèse ou synthèse de documents. Revue Pratiques, 79, 95-113.

Chervel, A. (2008). Histoire de l'enseignement du français du XVIIe au XXe siècle. Paris : Retz.

Chevallard, Y. (1991). La transposition didactique. Grenoble : la Pensée Sauvage.

Fontanille, J. (1989). Les espaces subjectifs. Paris : Hachette.

Kant, E. (2006). Critique de la raison pure (3 éd.). Paris : Flammarion.

Kristeva, J. (1978). Séméiôtiké. Paris : Seuil.

Mercier, A. (2004). Évaluer et comprendre les effets de l'enseignement sur les apprentissages des élèves: problématiques et méthodes en didactique des mathématiques et des sciences. Revue Française de Pédagogie, $148,47-59$.

Mercier, A. (à paraître). La didactique, une affaire de convention ? Le contrat didactique, convenances entre coutume et émergence. In J. Baillé (Ed.), Conventions. Grenoble: Presses Universitaires de Grenoble.

Nonnon, E. (2008). Travail des mots, travail de la culture et migration des émotions : les activités de français comme techniques sociales du sentiment. In M. Brossard § J. Fijalkow (Ed.), Vygotski et les recherches en éducation et en didactiques. Bordeaux : Presses universitaires de Bordeaux. 
Pérez, J.M. (2008). Approche comparative de l'enseignement des synthèses à l'Université : mise au jour d'un point aveugle. Lille : Atelier de reproduction des thèses.

Poucet, B. (2004). Les politiques éducatives en question. Paris : l'Harmattan.

Rochex, J. (1998). Le sens de l'expérience scolaire : entre activité et subjectivité. Paris : Presses Universitaires de France.

Verret, M. (1975). Le temps des études. Lille : Atelier de reproduction des thèses.

\section{TEXTES OFFICIELS}

Décret 72-355 du 4 mai 1972

Arrêté du 5 mars 1973 Modifié par le décret 2004-313 du 29 mars 2004 relatif aux conditions d'accès et aux régimes de formation à l'Ecole nationale d'administration.

Arrêté du 13 octobre 1999 fixant les programmes dans les épreuves de concours d'entrée à l'école Nationale de l'Administration.

Décret n 2004-313 du 29 mars 2004 modifiant le décret n 2002-50 du 10 janvier 2002 relatif aux conditions d'accès et aux régimes de formation à l'Ecole nationale d'administration.

Décret nº90-680 du 1 août 1990 relatif au statut particulier des professeurs des écoles.

Arrêté du 18 octobre 1991 modifié fixant les modalités d'organisation du concours externe, du concours externe spécial, du second concours interne, du second concours interne spécial et du troisième concours de recrutement de professeurs des écoles.

Note de service $\mathrm{n}^{\mathrm{O}}$ 94-271 du 16 Novembre 1994, modifiée par la note de service $\mathrm{n}^{\mathrm{O}}$ 95-017 du 19 janvier 1995 : Recommandations relatives aux concours de recrutement de professeurs des écoles.

Arrêté du 29 avril 2002 modifiant l'arrêté du 18 octobre 1991 fixant les modalités d'organisation du concours externe de recrutement de professeurs des écoles.

Arrêté du10 mai 2005, modalités d'organisation du concours externe, du concours externe spécial, du second concours interne, du second concours interne spécial et du troisième concours de recrutement de professeurs des écoles.

Note de service $\mathrm{n}^{\mathrm{O}}$ 2005-083du 16 mai 2005 relative au programmes permanents des concours externe, du concours externe spécial, du second concours interne, du second concours interne spécial et du troisième concours de recrutement de professeurs des écoles.

Bulletin Officiel n²1 du 26 mai 2005 : Modalités d'organisation du concours externe, du concours externe spécial, du second concours interne, du second concours interne spécial et du troisième concours de recrutement de professeurs des écoles. 